\title{
Effects of Amine-Functionalization on Mixed Matrix Membranes Encompassing of T-Type Zeolite and 6FDA-based Polyimide for Separation of $\mathrm{CO}_{2}$ from $\mathrm{CH}_{4}$
}

\author{
Norwahyu Jusoh, Y. F. Yeong*, K. K. Lau and A. M. Shariff \\ Chemical Engineering Department, Universiti Teknologi PETRONAS, \\ 32610 Bandar Seri Iskandar, Perak, Malaysia \\ *Email: yinfong.yeong@utp.edu.my \\ Phone: +6053687564; Fax: +6053656176
}

\begin{abstract}
Mixed matrix membranes (MMMs) comprising of unfunctionalized and aminefunctionalized T-type zeolite and 6FDA-based polyimide matrix were formulated. The physicochemical properties of the membranes were examined by FESEM, EDX, TGA and DSC. FESEM images showed that the enhancement of polymer-zeolite adhesion in MMM embedded with amine-functionalized T-type zeolite is trivial in comparison with MMM loaded with unfunctionalized T-type zeolite. From permeation test, permeability of $\mathrm{CO}_{2}$ of 858 Barrer and selectivity of $\mathrm{CO}_{2} / \mathrm{CH}_{4}$ of 22.5 was obtained using MMM incorporated with amine-functionalized T-type zeolite, whereas MMM incorporated with unfunctionalized T-type zeolite displayed permeability of $\mathrm{CO}_{2}$ of 844 Barrer and selectivity of $\mathrm{CO}_{2} / \mathrm{CH}_{4}$ of 19.1 . Thus, selection of another suitable aminefunctionalization group might be required in later works to further improve MMM performance in capturing $\mathrm{CO}_{2}$ from $\mathrm{CH}_{4}$.
\end{abstract}

Keywords: Mixed matrix membrane;6FDA-based polyimide; Amine-functionalized Ttype zeolite; $\mathrm{CO}_{2} / \mathrm{CH}_{4}$ separation.

\section{INTRODUCTION}

Over the past several decades, research in development of energy and $\mathrm{CO}_{2}$ capture techniques has been intensified mainly due to reduction of resource and global warming issues. Compared to conventional technologies including adsorption, absorption and cryogenic, membrane-based process has emerged due to its low space and weight requirement, low operating cost and environmental-friendly [1,2]. Polymeric membrane is primarily employed for a variety of industrial gas practices because of its advantages including inexpensive and easy to scale up. Nonetheless, these materials exhibit an extremity in the permeability/selectivity tradeoff as reported by Robeson [3]. Despite inorganic membrane displays relatively higher gas flux and selectivity as compared to polymeric membrane, it is restricted owing to high fabrication cost and hard to produce. Hence, this challenge demands the discovery of new materials with improved performance. Therefore, MMMs is introduced for the improvement of membrane performance by embedding dispersed solids including carbon molecular sieves and zeolites into polymer phase [4].

Zeolites have gained great attention in MMM fabrication because of their great molecular sieving property and narrow pore distribution [5]. T-type zeolites display a great prospective in separation of $\mathrm{CO}_{2}$ from $\mathrm{CH}_{4}$ owing to $0.36 \mathrm{~nm} \times 0.51 \mathrm{~nm}$ of aperture 
pores [6]. Therefore, small pores of 8-membered oxygen rings in T-type zeolites framework generate them as a potential filler in the formulation of MMM for separation of gases molecule and organic/aqueous mixtures [7, 8]. However, the formulation of MMMs with good compatibility and great polymer-filler adhesion remain challenging. Hence, several practices have been conducted to reinforce the polymer-filler adhesion and subsequently increase the separation performance. Aminosilane coupling agent is regularly utilized for the functionalization of filler properties, which bind the continuous and dispersed phases and consequently increase the adhesion properties between polymer and filler.

Mahajan and Koros [9] stated that polymer-filler adhesion of MMM loaded with silane-modified zeolite was improved although the reduction of permeability and selectivity in comparison with pure membrane was observed. The reduction of gas separation performance mainly contributed to the incapability to remove the interfacial voids in the membranes by silane coupling agent. Similar outcomes were also attained by Pechar et al. [10] as they combined APTES-functionalized ZSM-2 and polyimide matrix. In addition, Nik et al. [11] disclosed that the inclusion of amine-grafted MOF199 in 6FDA-ODA matrix enhanced the dispersion of particle in the polymer phase and subsequently elevate permeability of $\mathrm{CO}_{2}$ and selectivity of $\mathrm{CO}_{2} / \mathrm{CH}_{4}$ than that of $\mathrm{MMM}$ embedded with unfunctionalized MOF-199. In another work, Ahmad et al. [12] functionalized SAPO-44 by utilizing 3-aminopropyl-trimethoxysilane (APTMS). The amalgamation of functionalized SAPO-44 into polysulfone matrix improved gas pair selectivity more than $400 \%$ in comparison with MMM embedded with unfunctionalized SAPO-44. Overall, functionalized zeolite has increased the polymer-filler adhesion properties and consequently improved the membrane performance.

To the author knowledge, the investigation on the formation of MMM incorporated with amine-functionalized T-type zeolite for capturing of $\mathrm{CO}_{2}$ from $\mathrm{CH}_{4}$ has not been discovered so far. Thus, in this work, 3-(2-(2-aminoethylamino) ethylamino) propyltrimethoxysilane (AEPTMS) silane coupling agent was used to functionalize T-type zeolite prior to load in 6FDA-based polyimide matrix. The physicochemical properties of the fabricated membranes were then measured by utilizing various analytical instruments. Furthermore, permeation properties of aminefunctionalized T-type zeolite/6FDA-based polyimide MMM was investigated and measured the differences with unfunctionalized T-type zeolite loaded MMM.

\section{EXPERIMENTAL SETUP}

\section{Chemicals and Gases}

T-type zeolite crystals and 6FDA-based polyimide polyimide were prepared by our research laboratory. Materials for the synthesis of amine-functionalized T-type zeolite crystals require 3-(2-(2-aminoethylamino) ethylamino) propyltrimethoxysilane (AEPTMS, $\mathrm{C}_{10} \mathrm{H}_{27} \mathrm{~N}_{3} \mathrm{O}_{3} \mathrm{Si}$, Sigma Aldrich) and toluene $\left(\mathrm{C}_{6} \mathrm{H}_{5} \mathrm{CH}_{3}\right.$, Sigma Aldrich). Materials for producing membranes including dichloromethane (DCM) were obtained from Sigma Aldrich. Furthermore, for membrane gas permeation, the $99.9995 \%$ purified carbon dioxide and methane gases were provided by Gas Walker. 


\section{Synthesis of T-type Zeolite and Amine-functionalized T-type zeolite Crystals}

T-type zeolite crystals was prepared with molar composition of $\mathrm{SiO}_{2}: 0.04 \mathrm{Al}_{2} \mathrm{O}_{3}$ : $0.26 \mathrm{Na}_{2} \mathrm{O}: 0.09 \mathrm{~K}_{2} \mathrm{O}: 14 \mathrm{H}_{2} \mathrm{O}$ by method of sonochemical-assisted hydrothermal growth [6]. Meanwhile, amine-functionalized T-type zeolite was synthesized via the technique practiced by Li et al. [12]. T-type zeolite crystals, toluene and AEPTMS aminosilane coupling agent were blended and agitated for $24 \mathrm{~h}$ under nitrogen environment. The solid crystals were collected by centrifugation prior to wash with methanol and dry at $110^{\circ} \mathrm{C}$ in an oven for $24 \mathrm{~h}$.

\section{Formation of Pure 6FDA-based Polyimide and Mixed Matrix Membranes}

Pure 6FDA-based polyimide film was formed according to our reported method [6]. A 6FDA-based polyimide solution was cast onto glass Petri dish prior to dry and anneal at $250^{\circ} \mathrm{C}$ for $24 \mathrm{~h}$ under vacuum condition. Meanwhile, MMMs incorporated with $1 \mathrm{wt} \%$ of unfunctionalized and functionalized T-type zeolite particles were prepared via method of solution blending [8]. A slurry comprising of 6FDA-based polyimide and Ttype zeolite was blended, followed by casting onto glass Petri dish. The membrane was dried before annealing for $24 \mathrm{~h}$.

\section{Membranes Characterization}

The fabricated membranes were characterized by field emission scanning electron microscopy (FESEM), energy dispersive X-ray (EDX) spectroscopy, thermal gravimetric analysis (TGA) and differential scanning calorimetry (DSC). The morphology of membrane was attained using Zeiss Supra 55 VP FESEM. The sputter coater (Quorum Q150R S) was used to sputter coat the samples with platinum target before imaging analysis. In addition, EDX (Oxford Instrument Inca mapping) was utilized to examine the particle dispersion in the continuous phase. The thermal stability of crystals and membranes were obtained by TGA (Perkin Elmer STA6000) under air environment up to $800^{\circ} \mathrm{C}$ at the heating rate of $10^{\circ} \mathrm{Cmin}^{-1}$. On the other hand, the glass transition temperature $\left(\mathrm{T}_{\mathrm{g}}\right.$ ) of MMMs was examined with DSC (DSC Q2000, Thermal Analysis (TA)). The $\mathrm{T}_{\mathrm{g}}$ of the membranes were obtained from the second heating cycle.

\section{Permeation Tests}

Gas permeation properties were determined by permeation test rig at 3.5 bar and $30^{\circ} \mathrm{C}$ for $\mathrm{CO}_{2}$ and $\mathrm{CH}_{4}$ gases. The detailed description on the testing operation and technique were provided elsewhere [1]. The permeability of each gas was attained using Eq. (1) as follows [13]:

$$
P=\frac{V_{p} t}{A_{m}\left(p_{h}-p_{l}\right)}
$$

where $P$ designates the membrane permeability (Barrer) (1 Barrer $=1 \times 10^{-10}$ $\left.\mathrm{cm}^{3}(\mathrm{STP}) . \mathrm{cm} / \mathrm{s} . \mathrm{cm}^{2} . \mathrm{cmHg}\right), V_{p}$ refers to the flow rate of permeate $\left(\mathrm{cm}^{3}(\mathrm{STP}) / \mathrm{s}\right), t$ is the thickness of membrane $(\mathrm{cm}), A_{m}$ refers to the area of membrane $\left(\mathrm{cm}^{2}\right), p_{h}$ and $p_{l}$ are the 
pressure of feed and permeate, respectively $(\mathrm{cmHg})$. The membrane selectivity was obtained using Eq. (2) as follows [14].

$$
\alpha_{\mathrm{CO}_{2} / \mathrm{CH}_{4}}=\frac{P_{\mathrm{CO}_{2}}}{P_{\mathrm{CH}_{4}}}
$$

where $\alpha$ is the membrane ideal selectivity.

\section{RESULTS AND DISCUSSION}

\section{Membranes Characterization}

The morphology of the MMMs loaded with unfunctionalized and amine functionalized T-type zeolite is demonstrated in Figure 1. Referring to Figure 1 (b) and (c), it can be seen that both of the MMMs display well distribution of T-type zeolite particles in 6FDA-based polyimide phase without any interfacial voids between the polymer and particle. This signifies that a great compatibility between 6FDA-based polyimide and Ttype zeolite has been achieved [14].

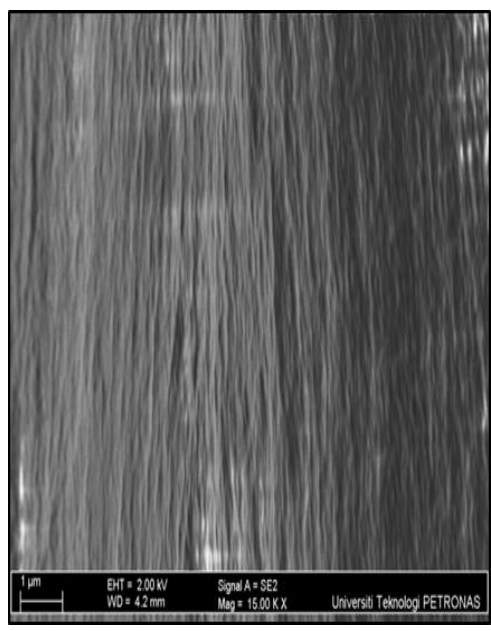

(a)

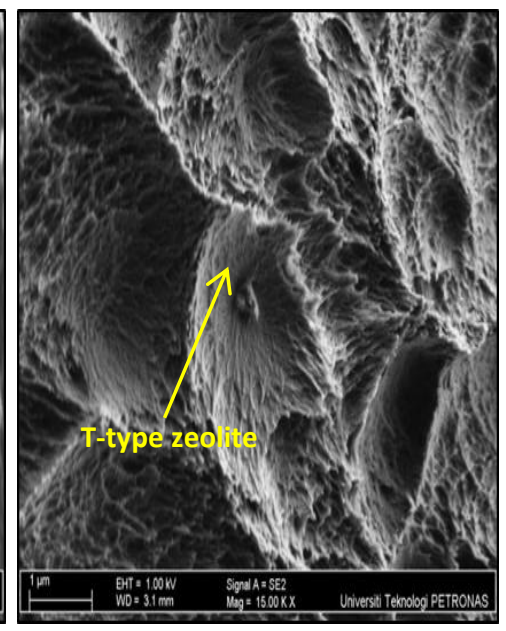

(b)

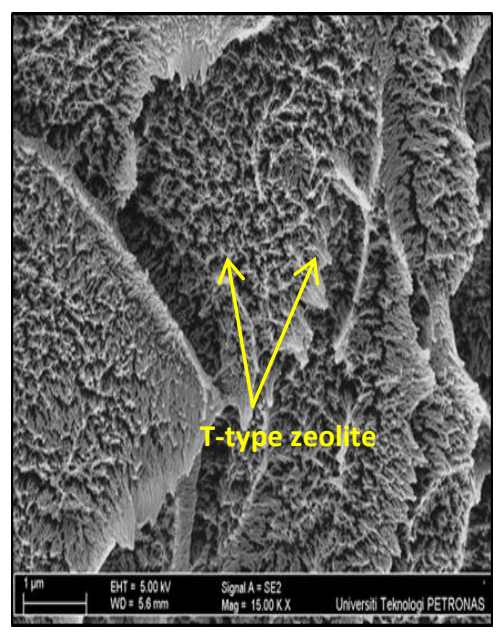

(c)

Figure 1. Morphologies of (a) pure 6FDA-based polyimide membrane (b) unfunctionalized T-type zeolite/6FDA-based polyimide and (c) aminefunctionalized T-type zeolite/6FDA-based polyimide MMMs.

A good particles dispersion in MMM incorporated with unfunctionalized and amine-functionalized T-type zeolite are further confirmed via EDX-mapping images displayed in Figure 2 (a) and (b), accordingly. Hence, unsubstantial effect on the polymer-filler adhesion properties was found when amine-functionalized T-type zeolite particles were loaded into 6FDA-based polyimide matrix. This observation might be due to the insignificant changes of microvoids formation and chain packing of membrane matrix after the incorporation of amine-functionalized T-type zeolite than that of unfunctionalized T-type zeolite loaded MMM $[12,15]$. 


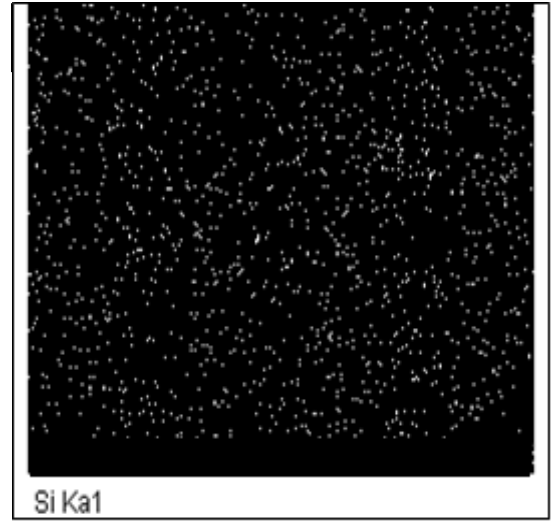

(a)

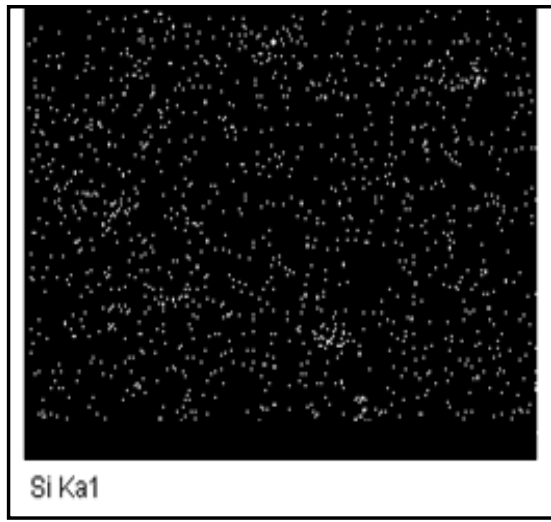

(b)

Figure 2. EDX-mapping images from the cross-section of MMM loaded with (a) unfunctionalized T-type zeolite and (b) amine-functionalized T-type zeolite.

The thermal stability of the particles and membranes are demonstrated in Figure 3. Based on Figure 3, the total weight loss for unfunctionalized and aminefunctionalized T-type zeolite particles were $14.9 \mathrm{wt} \%$ and $24.8 \mathrm{wt} \%$, respectively. The enhancement of the weight loss of amine-functionalized T-type zeolite than that of the unfunctionalized T-type zeolite probably because of the substantial interaction between T-type zeolite surface and amine functional group, where the covalent bond interaction was occurred. Meanwhile, the pure 6FDA-based polyimide reveals thermal stability up to $\sim 500{ }^{\circ} \mathrm{C}$, which is in accordance with reported literature [13]. In addition, based on Figure 3, all MMMs indicate stability of thermal around $500{ }^{\circ} \mathrm{C}$. It also can be seen that the amalgamation of amine-functionalized T-type zeolite in 6FDA-based polyimide declines the degradation temperature. This behavior might be contributed from weight loss enhancement of amine-functionalized T-type zeolite in comparison with unfunctionalized T-type zeolite particles $[16,17]$. Overall, all the fabricated MMMs demonstrate trivial variations in the weight residues, mainly because of the similar filling of unfunctionalized and amine-functionalized T-type zeolite $(1 \mathrm{wt} \%)$ loaded in 6FDA-based polyimide matrix for all MMMs [18].

Table 1 displays the $\mathrm{T}_{\mathrm{g}}$ of the resultant pure 6FDA-based polyimide and MMMs. Based on Table 1, pure 6FDA-based polyimide and unfunctionalized and aminefunctionalized T-type zeolite/6FDA-based polyimide MMM exhibit $\mathrm{T}_{\mathrm{g}}$ of $410.06{ }^{\circ} \mathrm{C}$, $425.22{ }^{\circ} \mathrm{C}$ and $425.53{ }^{\circ} \mathrm{C}$, respectively. Therefore, the amalgamation of aminefunctionalized T-type zeolite in 6FDA-based polyimide demonstrates trivial changes in the structure of polymer packing and membrane rigidity than those MMM embedded with unfunctionalized T-type zeolite [19]. 


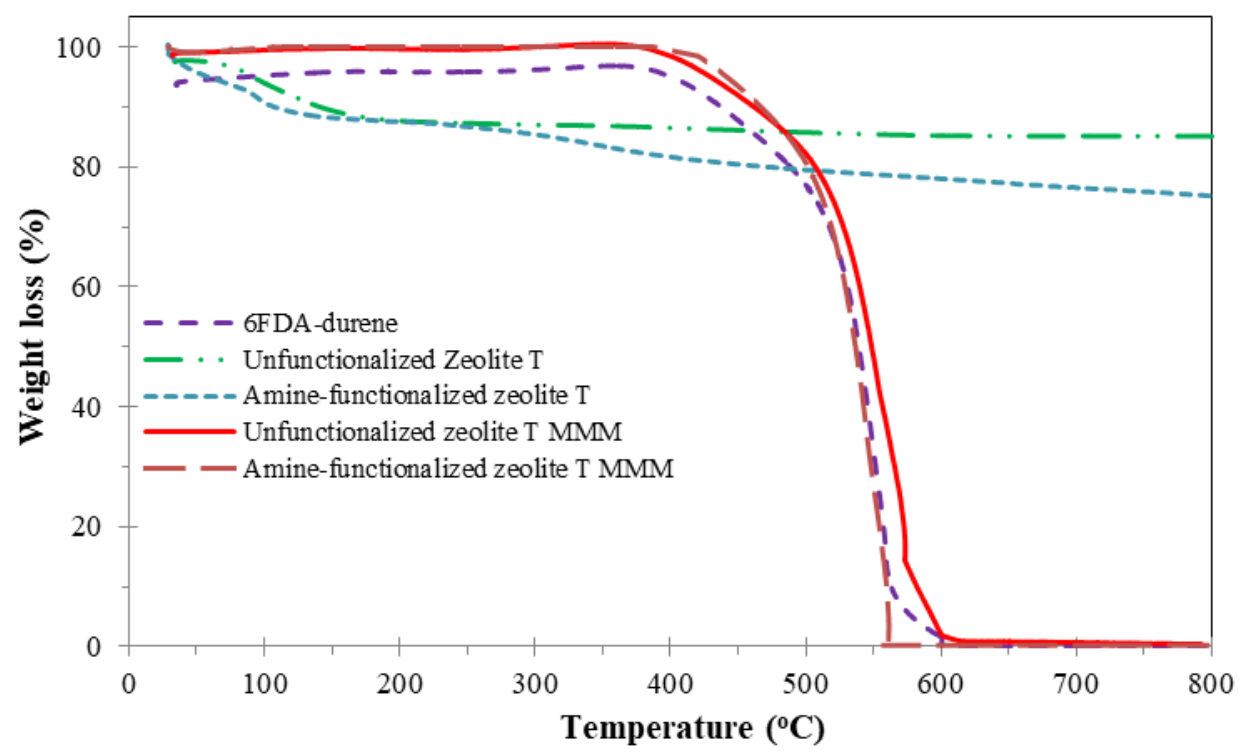

Figure 3. Thermal stability of the crystals and the membranes formulated in the current research.

Table $1 . T_{g}$ of the resultant membranes formed in the current research

\begin{tabular}{ll}
\hline \multicolumn{1}{c}{ Membrane } & $\mathrm{T}_{\mathrm{g}}\left({ }^{\circ} \mathrm{C}\right)$ \\
\hline Pure 6FDA-based polyimide & 410.06 \\
MMM incorporated with unfunctionalized T-type & 425.22 \\
zeolite & \\
$\begin{array}{l}\text { MMM incorporated with amine-functionalized T- } \\
\text { type zeolite }\end{array}$ & 425.53 \\
\hline
\end{tabular}

\section{Membrane Performance}

Performance of the pure 6FDA-based polyimide and MMMs in gas separation was examined by utilizing $\mathrm{CO}_{2}$ and $\mathrm{CH}_{4}$ pure gases permeation tests. Figure 4 demonstrates the permeability of $\mathrm{CO}_{2}$ and $\mathrm{CH}_{4}$ and selectivity of $\mathrm{CO}_{2} / \mathrm{CH}_{4}$ of the resultant membranes. According to Figure 4, both MMMs exhibit greater permeability of $\mathrm{CO}_{2}$ and selectivity of $\mathrm{CO}_{2} / \mathrm{CH}_{4}$ than pure $6 \mathrm{FDA}$-based polyimide membrane. Meanwhile, MMMs display lower $\mathrm{CH}_{4}$ permeabilities than that of pure 6FDA-based polyimide membrane.

The enhancement of MMMs gas separation performance in comparison with pure 6FDA-based polyimide could be accredited to the interruption of the packing of polymer chain by the existence of T-type zeolite in 6FDA-based polyimide matrix which subsequently improves the membrane free volume [20, 21]. In addition, the remarkable improvement of gas pair selectivity with the amalgamation of T-type zeolite (unfunctionalized and amine-functionalized) mainly contributed from the adsorption and sieving effects of T-type zeolite [7, 22]. Furthermore, favorable interaction between T-type zeolite and $\mathrm{CO}_{2}$ could aid the transport of gases through the membrane $[14,15]$.

On the other hand, MMM containing amine-functionalized T-type zeolite particles shows slight enhancements of permeability of $\mathrm{CO}_{2}$ of 858 Barrer and selectivity of $\mathrm{CO}_{2} / \mathrm{CH}_{4}$ of 22.5 than that of MMM loaded with unfunctionalized T-type 
zeolite with permeability of $\mathrm{CO}_{2}$ of 844 Barrer and selectivity of $\mathrm{CO}_{2} / \mathrm{CH}_{4}$ of 19.1 . The negligible increment of permeability of $\mathrm{CO}_{2}$ and selectivity of $\mathrm{CO}_{2} / \mathrm{CH}_{4}$ of $\mathrm{MMM}$ embedded with amine-functionalized T-type zeolite mainly because of the insignificant changes of polymer-filler interfacial adhesion than that of the MMM embedded with unfunctionalized T-type zeolite particles as supported by FESEM and EDX-mapping images $[23,24]$. In addition, as shown in Table 1 , no significant changes of $\mathrm{T}_{\mathrm{g}}$ of this membrane in comparison with T-type zeolite/6FDA-based polyimide MMM also validates minor enhancement of membrane separation performance.

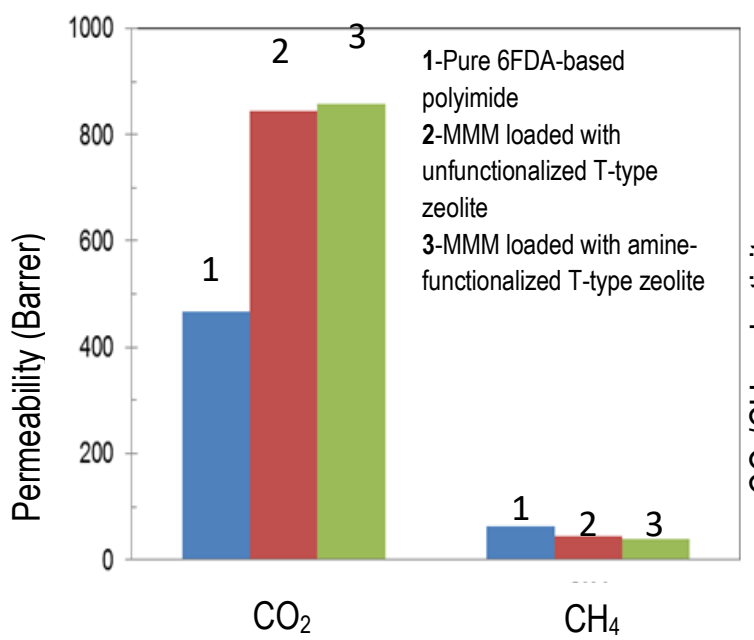

(a)

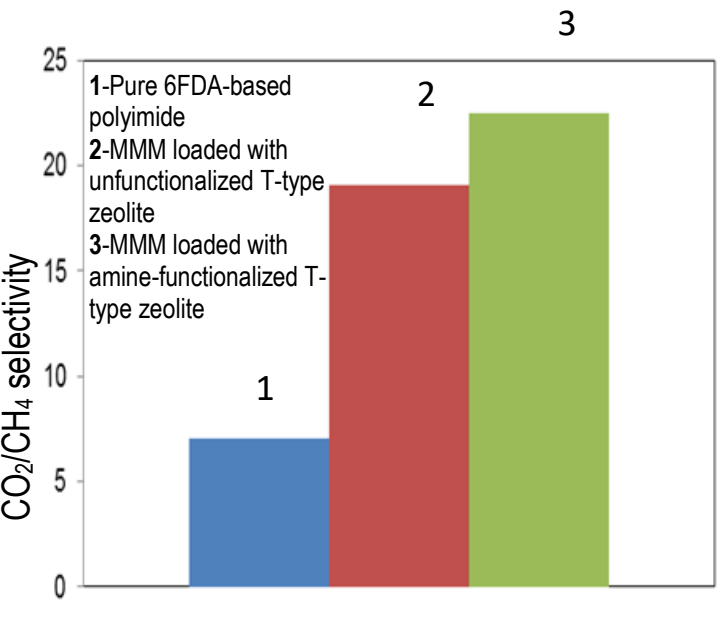

(b)

Figure 4. (a) Permeabilities of $\mathrm{CO}_{2}$ and $\mathrm{CH}_{4}$ and;(b) selectivities of $\mathrm{CO}_{2} / \mathrm{CH}_{4}$ of the resultant membranes formed in the current work.

\section{CONCLUSION}

In this work, MMM embedded with unfunctionalized and amine-functionalized T-type zeolite particles have been fabricated. From FESEM images, MMM incorporated with amine-functionalized T-type zeolite displayed insignificant enhancement on the interfacial adhesion between polymer and particles phase than those MMM containing unfunctionalized T-type zeolite particles. Based on gas permeation results, the combination of amine-functionalized T-type zeolite and 6FDA-based polyimide slightly improved permeability of $\mathrm{CO}_{2}$ and selectivity of $\mathrm{CO}_{2} / \mathrm{CH}_{4}$ in comparison unfunctionalized T-type zeolite/6FDA-based polyimide MMM. Therefore, selection of another suitable amine-functionalization group might be required in future works in order to further increase the performance of $\mathrm{MMM}$ in capturing $\mathrm{CO}_{2}$ from $\mathrm{CH}_{4}$.

\section{ACKNOWLEDGEMENT}

The authors would like to acknowledge $\mathrm{CO}_{2}$ Research Centre $\left(\mathrm{CO}_{2} \mathrm{RES}, 015 \mathrm{NEC}-001\right)$, Universiti Teknologi PETRONAS for supports. 


\section{REFERENCES}

[1] Jusoh N, Lau KK, Shariff AM, Yeong YF. Capture of bulk CO2 from methane with the presence of heavy hydrocarbon using membrane process. International Journal of Greenhouse Gas Control. 2014;22:213-22.

[2] Zakaria I, Michael Z, Mohamed WANW, Mamat AMI, Azmi WH, Mamat R, et al. A review of nanofluid adoption in polymer electrolyte membrane (PEM) fuel cells as an alternative coolant. Journal of Mechanical Engineering and Sciences. 2015;8:1351-66.

[3] Liu C, Kulprathipanja S, Hillock AMW, Husain S, Koros WJ. Recent Progress in Mixed-Matrix Membranes. Advanced Membrane Technology and Applications: John Wiley \& Sons, Inc.; 2008. p. 787-819.

[4] Wiryotmojo AS, Mukhtar H, Man Z. Development of polysulfone carbon molecular sieves mixed matrix membranes for $\mathrm{CO} 2$ removal from natural gas. International Conference on Chemical, Biological and Environemntal Engineering. Singapore2009.

[5] Adams R, Carson C, Ward J, Tannenbaum R, Koros W. Metal organic framework mixed matrix membranes for gas separations. Microporous and Mesoporous Materials. 2010;131:13-20.

[6] Jusoh N, Yeong YF, Mohamad M, Lau KK, M. Shariff A. Rapid-synthesis of zeolite $\mathrm{T}$ via sonochemical-assisted hydrothermal growth method. Ultrasonics Sonochemistry. 2017;34:273-80.

[7] Veerapur RS, Patil MB, Gudasi KB, Aminabhavi TM. Poly(vinyl alcohol)zeolite $\mathrm{T}$ mixed matrix composite membranes for pervaporation separation of water + 1,4-dioxane mixtures. Separation and Purification Technology. 2008;58:377-85.

[8] Jusoh N, Yeong YF, Lau KK, M. Shariff A. Enhanced gas separation performance using mixed matrix membranes containing zeolite $\mathrm{T}$ and 6FDAdurene polyimide. Journal of Membrane Science. 2017;525:175-86.

[9] Pechar TW, Tsapatsis M, Marand E, Davis R. Preparation and characterization of a glassy fluorinated polyimide zeolite-mixed matrix membrane. Desalination. 2002;146:3-9.

[10] Clarizia G, Algieri C, Regina A, Drioli E. Zeolite-based composite PEEK-WC membranes: Gas transport and surface properties. Microporous and Mesoporous Materials. 2008;115:67-74.

[11] Nik OG, Chen XY, Kaliaguine S. Functionalized metal organic frameworkpolyimide mixed matrix membranes for $\mathrm{CO} 2 / \mathrm{CH} 4$ separation. J Membrane Sci. 2012;413-414:48-61.

[12] Li Y, Guan H-M, Chung T-S, Kulprathipanja S. Effects of novel silane modification of zeolite surface on polymer chain rigidification and partial pore blockage in polyethersulfone (PES)-zeolite A mixed matrix membranes. Journal of Membrane Science. 2006;275:17-28.

[13] Mohammadi T, Moghadam MT, Saeidi M, Mahdyarfar M. Acid Gas Permeation Behavior Through Poly(Ester Urethane Urea) Membrane. Ind Eng Chem Res. 2008;47:7361-7.

[14] Jusoh N, Yeong YF, Cheong WL, Lau KK, M. Shariff A. Facile fabrication of mixed matrix membranes containing 6FDA-durene polyimide and ZIF-8 
nanofillers for $\mathrm{CO} 2$ capture. Journal of Industrial and Engineering Chemistry. 2016;44:164-73.

[15] Recio R, Lozano ÁE, Prádanos P, Marcos Á, Tejerina F, Hernández A. Effect of fractional free volume and $\mathrm{Tg}$ on gas separation through membranes made with different glassy polymers. Journal of Applied Polymer Science. 2008;107:103946.

[16] Northcott KA, Bacus J, Taya N, Komatsu Y, Perera JM, Stevens GW. Synthesis and characterization of hydrophobic zeolite for the treatment of hydrocarbon contaminated ground water. Journal of Hazardous Materials. 2010;183:434-40.

[17] Mohamed WANW, Atan R. Analysis of excessive heating on the thermal and electrical resistance of a polymer electrolyte membrane fuel cell. International Journal of Automotive and Mechanical Engineering. 2012;5:648-59.

[18] Rahmat N, Yusof NFM, Hafiza E. Thermogravimetric analysis (TGA) profile of modified SBA-15 at different amount of alkoxysilane group. The Malaysian Journal of Analytical Sciences. 2014;18:730-6.

[19] Mahajan R, Burns R, Schaeffer M, Koros WJ. Challenges in forming successful mixed matrix membranes with rigid polymeric materials. Journal of Applied Polymer Science. 2002;86:881-90.

[20] Askari M, Chung T-S. Natural gas purification and olefin/paraffin separation using thermal cross-linkable co-polyimide/ZIF-8 mixed matrix membranes. Journal of Membrane Science. 2013;444:173-83.

[21] Yeong YF, Wang H, Pallathadka Pramoda K, Chung T-S. Thermal induced structural rearrangement of cardo-copolybenzoxazole membranes for enhanced gas transport properties. Journal of Membrane Science. 2012;397-398:51-65.

[22] Chen X, Wang J, Yin D, Yang J, Lu J, Zhang Y, et al. High-performance zeolite $\mathrm{T}$ membrane for dehydration of organics by a new varying temperature hot-dip coating method. AIChE Journal. 2013;59:936-47.

[23] Mahajan R, Koros WJ. Mixed matrix membrane materials with glassy polymers. Part 1. Polymer Engineering \& Science. 2002;42:1420-31.

[24] Mahajan R, Koros WJ. Mixed matrix membrane materials with glassy polymers. Part 2. Polymer Engineering \& Science. 2002;42:1432-41. 